\title{
Automatic Vertebral Body Fracture Classification through PCA based Angle Estimation
}

\author{
Adela Arpitha" ${ }^{\# 1}$, Lalitha Rangarajan" ${ }^{\# 1}$, Raghunandan K. S \#1 \\ ${ }^{\# 1}$ Department of Studies in Computer Science, University of Mysore, Mysuru - 570006 \\ adelaarpitha23@gmail.com, lali85arun@yahoo.co.in, raghu0770@gmail.com
}

\section{ABSTRACT}

Our objective is to contribute a reliable platform for segmenting and classifying vertebral fractures. We present a method for the automatic segmentation of the Vertebral Bodies (VB) from 2D sagittal images of the spine followed by classifying if the VB is normal or fractured. In our work, we use Dual energy X-ray Absorptiometry (DXA) images which is a standard technique to quantify Bone Mineral Density (BMD) of vertebrae that best indicates osteoporotic vertebral fracture. A certain level of preprocessing is required as the DXA images appear noisy. After image binarization, morphological operations are applied to close the missing links and to remove small invalid regions. The Region of Interest (ROI) is portioned by extricating fine contour of vertebral endplates. The count of Connected Components (CC) indicates the presence of inferior and superior endplates of the adjacent vertebrae. A complete VB is segmented by choosing those two CCs with the largest centroid distance. Principal Component Analysis (PCA) inspired angle estimation for each CC is used to classify if the segmented VB is fractured or not. Our approach has been demonstrated to be feasible both in terms of efficiency and robustness by being tested on 527 vertebrae on DXA images at a classification accuracy of $93.93 \%$ compared to a gold standard of manual annotation by expert radiologists.

Key words: Automatic segmentation, Classification, Morphology, Connected components, PCA angle, DXA

Corresponding Author: Adela Arpitha

\section{INTRODUCTION}

Image data is of immense practical importance in medical informatics. A fundamental objective of medical image processing is to transform raw images into digital form for enhanced representation, evaluation, and furthermore for content based search and mining [1]. Medical image processing enables the clinicians to see fine points of interest of the images that are hard to recognize by naked eyes [2].

A fundamental step in this transformation is the segmentation of the objective structures that depends on the given homogeneity criteria. The job is to separate the image into regions, which, in medical images, are typically the objective anatomic regions (foreground) and their neighborhood (background).After this division, the specific shape and appearance features of the objectives can be computed, and, in light of the application, they can be utilized for clinical assessment, analyzing patterns, and additional information discovery. [1]. With growing use of Computed Tomography (CT) and Magnetic Resonance Imaging (MRI) and other modalities for diagnosis, treatment, medication planning, and clinical examinations, it 
has turned out to be practically necessary to utilize PCs to help radiological specialists. Autonomous diagnosis frameworks helps in handling large number of cases with similar precision, accomplishes quick and exact outcomes at moderate expenses that can help the specialist in inspecting the bone fitness, which goes about as a second opinion. Generally, for a minor fracture, it may go undetected by the doctor or specialist, thus prompting a wrong diagnosis [3].

The spine life system in figure 1, can be depicted as a weight bearing hard portioned skeletal structure made up of 33 bones called vertebrae separated into 5 areas in particular cervical -7 vertebrae, thoracic -12 vertebrae, lumbar -5 vertebrae, sacrum -5 vertebrae and coccyx -4 vertebrae that are stacked over each other with ductile intervertebral plates in the middle of every vertebra. A vertebra comprises of two sections: an anterior portion that forms the VB and a posterior or the vertebral bend that comprises of spinous procedures, pedicles, vertebral lower arm and other anatomical locales [4].

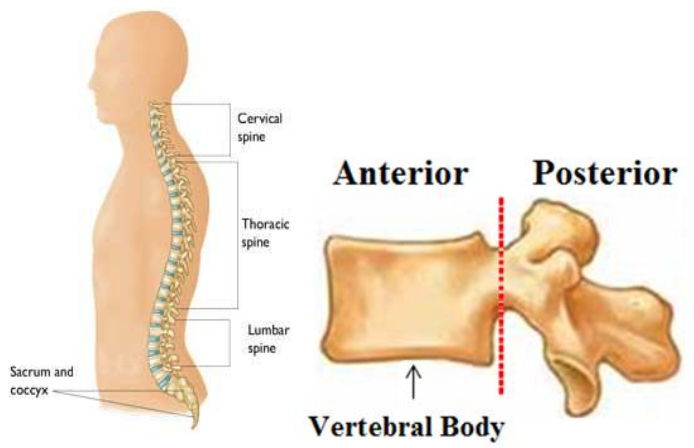

Fig 1. Anatomy of a human vertebra

Spine-related ailments and conditions, for example, degenerative disc disease, osteoporosis, scoliosis, spinal stenosis, herniated discs, fractures, ligamentous damage, tumor, infection, and spondyloarthropathy are common, causing immense discomfort and additional cost to the society. Medications differ with the conditions, and the clinical situation can be nonspecific. Thus, imaging is regularly required to help make the diagnosis and studies easier by incorporating plain radiographs, DXA, CT, MRI, PET, bone scans, ultrasound (US), and nuclear medicine. Computational strategies are relentlessly booming to enhance speed, certainty, and precision in achieving a final diagnosis. [5]

Segmentation is by and large the primary stage in a bid to examine or describe an image automatically. The success or failure of an assignment is a straightforward result of the success or failure of the segmentation. Nonetheless, an accurate and reliable segmentation of an image, as a rule, is extremely hard to accomplish by completely automatic means. DXA pictures captured for Vertebral Fracture Assessment (VFA) commonly cover the spine anatomy from T4 to L4 (T-Thoracic, L-Lumbar). Accurate and robust segmentation of vertebral structures is challenging because the acquired images often have deficient boundaries or missing edges. Furthermore the absence of texture contrast in the midst of regions of interest (ROI) and background [1], a high level of anatomical complexity, significant irregular and structural noise alongside such as overlapping bony structures (ribs, scapulae and iliac crests) and delicate tissues, low signal-to-noise ratio, low spatial resolution, and non-homogeneity [6] complicates the segmentation task. The segmentation performance further goes down when the images are impaired by artifacts, various outliers and number of detectable vertebrae. It is required to reduce the noise before extracting the edges as it affects the performance of the further steps like segmentation and classification. 
DOI: https://dx.doi.org/10.26808/rs.ca.i8v1.19

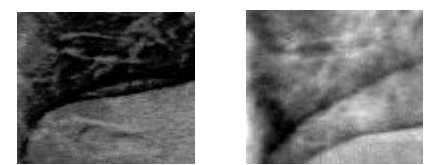

Fig 2. Disparity of sagittal lumbar DXA images

To address these issues, a couple of vertebral body segmentation approaches on various modalities have been proposed in the literature. However, many of these methods assume that the number of vertebrae visible in the image is known a priori. This is a significant limitation. The dominantly used traditional segmentation procedures, thresholding approaches, semiautomatic methods [7] encounter restraints because of considerable dependence on user interaction. Common vulnerabilities in an image cannot be captured by these approaches. Familiarity with vertebra location, shape and direction is noteworthy in various medical applications. [8] has handled this issue without making use of any shape priors by a two-step strategy where at first a coarse-to-fine system conveys a coarse condition of the vertebra. At that point, segmentation is refined by a Hidden Markov Chain (HMC) segmentation using a specific volume transformation. Several authors have investigated the use of methods based on statistical shape models [9] and Active Appearance Models (AAMs), [10] to segment vertebrae in DXA images as a preliminary step for VFA. However, state-of-the-art results achieved using AAMs exhibit significant numbers of large errors due to fit failures and the generalizability is poor due to single model of shape and intensity that covers all landmarks.

Several authors have proposed solutions to the more general problem of vertebra localization where the number present is not known a priori. In [11], a framework is described for fully automatic localization, identification and segmentation of vertebrae in CT images, by producing a triangulated surface mesh and a level label for all visible vertebrae. However, a complex chain of processing steps is required.

Despite its clinical concern, automatic accurate classification of vertebral fractures is difficult and tedious. Various attempts have been made to automate the procedure using different heuristics yet with restricted achievement. Literature lists a considerable number of automatic methods for the assessment of vertebral fractures from DXA images [10], [12]. Many of the methods proposed are based on Algorithm Based Qualitative (ABQ) technique [13] that well differentiates vertebral fractures from deformities, which is ideally suited for certain type of vertebral fractures and specific datasets. However, the ground truth doesn't generally work with the state of the art technique.

The most generally known osteoporotic fracture is the vertebral fracture. Many a times, this result from routine activities, for instance, turning, twisting, or lifting light things, and are also associated with falls [14]. The decision of vertebral fracture can challenge and to some extent be arguable to be proven wrong as there is no absolute "gold standard" for the judgment of vertebral fracture [15]. Presently, the highest quality level approach for the recognition of vertebral fractures is through visual examination.

A primal to visually distinguish fractured and healthy vertebrae is a thorough know-how of the normal range and abnormality in shape and appearance of vertebral endplates. Vertebral fractures more often than not cause change of the vertebral shape. Thus, it is imperative to attentively examine the vertebral shape and endplate features to discriminate healthy vertebrae from the fractured ones [16]. There is profound proof that vertebral fractures are under-reported and when exists, appropriate intervention may not occur. In general, radiology reports one out of three vertebral fractures being misdiagnosed ( $34 \%$ false negatives), subsequent to some extent as a lack of radiographic recognition or use of ambiguous remarks in reports. Hence, there is need for clear and unambiguous reporting of exact diagnosis [17]. 
Since a substantial percentage of vertebral fractures do not come to clinical attention, imaging is believed to be an optimal manner in clinical practice to identify and confirm the presence of osteoporotic vertebral fractures. Vertebral fracture analysis requires a highly accurate per vertebra region of interest (ROI) fit because fractures are correlated with the height of the vertebra [10]. The variation in height alone cannot be used as a measure to distinguish vertebral fractures. It may be caused because of different reasons like deformities or diseases or deceiving appearance as a result of imaging artifacts. Visual hints such as parallelism of the endplates and absence of structural horizontal straightness and nonappearance of homogeneity with neighboring vertebrae used by specialists to determine the qualities of the fractures ought to be utilized. Therefore, it is useful to develop quantitative approaches which include such observations of the radiologists and perhaps conflicting opinions of radiologists could be avoided.

Our aim in this work is to develop an image analysis approach competent enough to analyze and classify vertebral fractures from traditional VFA DXA information that stands as a solid preliminary step before categorizing various types and grades of fractures. In the light of this inspiration, given a DXA dataset of the spine, our method preprocesses and segments six thoracic vertebrae ( $\mathrm{T} 7$ to $\mathrm{T} 12$ ) and four lumbar vertebrae and classifies if the vertebra is normal or fractured.

\section{PROPOSED METHODOLOGY}

The work proposed in this paper is segmentation of endplates of VB for the purpose of determination of fractures of VB. The sequence of steps is as given in figure 3 .

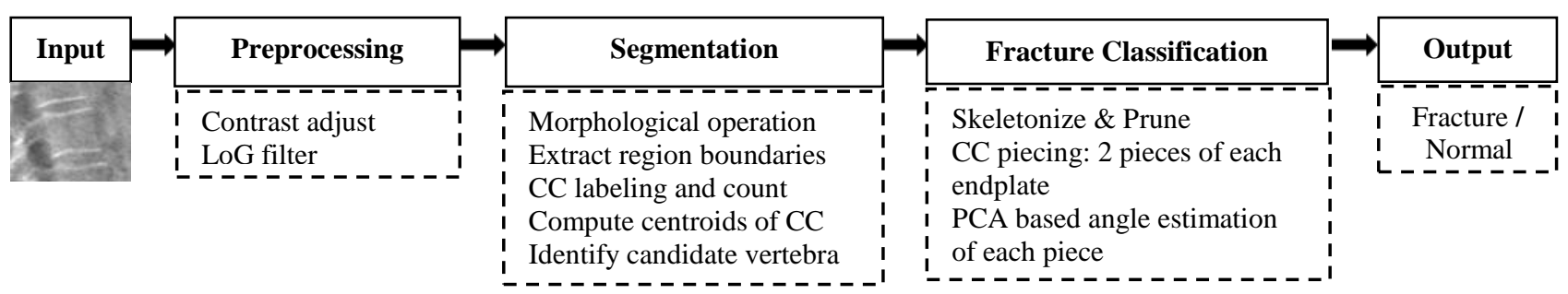

Fig 3. Proposed methodology for segmentation and classification of vertebral fractures

The dataset needs preprocessing as the images contain differing noise levels. Amplification of the contrast of the image will expand the accuracy of the ensuing modules for an autonomous fracture diagnosis framework. Thus, the input image is enhanced by first adjusting the contrast to blend with the logarithmic filter which works well in overcoming the differing noise levels effectively present in DXA images.

Once we have the noise reduced image, we binarize the image by a thresholding factor ' $\mathrm{T}$ ' (1) that signifies the bony structures.

$$
\mathrm{I}(\mathrm{x}, \mathrm{y})= \begin{cases}1, & \text { if } \mathrm{I}(\mathrm{x}, \mathrm{y})>\mathrm{T} \\ 0, & \text { otherwise }\end{cases}
$$

The image is further refined by applying relevant morphological operations. To cap small discontinuities, morphological closing is done with a structuring element ' $C$ ' $(2)$ and morphological opening to remove small invalid regions lesser than a threshold ' $\mathrm{O}$ ' is done using (3).

$$
\begin{aligned}
& \mathrm{ROI} \bullet \mathrm{C}=(\mathrm{ROI} \oplus \mathrm{C}) \ominus \mathrm{C} \\
& \mathrm{ROI} \bullet \mathrm{O}=(\mathrm{ROI} \oplus \mathrm{O}) \oplus \mathrm{O}
\end{aligned}
$$


The ROI is portioned by extracting fine boundaries of the vertebrae. The dataset is available in the following format: a single spine is cropped into individual vertebra from T7 to L5. Each individual cropped image of the vertebra also contains the (presence of) inferior endplate of the vertebra above and the superior endplate of the vertebra below it as shown in figure 4 (a).

Based on the prior knowledge that osteoporotic fractures are almost closely endplate fractures [18], we try to carefully segment the endplates of one complete vertebra by removing the half vertebral endplates. In order to segment one complete vertebra from the ROI, the number of $\mathrm{CC}$ are first counted and labeled. Next, the centroid (4) of each CC is located and then the adjacent pairwise distance ratio for all labeled adjacent pairs of centroid is found (5).

$$
\begin{gathered}
\left\{\mathrm{C}\left(\mathrm{x}_{\mathrm{i}}, \mathrm{y}_{\mathrm{i}}\right) \mid \mathrm{i}=1: 4\right\} \\
\mathrm{d}_{\mathrm{i}}=\sqrt{\left(x_{i}-x_{j}\right)+\left(y_{i}-j\right)^{2}} \mid \mathrm{i}, \mathrm{j}=1: 4, \mathrm{i}>\mathrm{j}
\end{gathered}
$$

Sorting of the distances in descending order shows the pair of CC that has the second largest distance between them. That pair of CC is retained as the candidate vertebra.

The bounding box for the relevant pair of CC completes the segmentation task as in figure 4 (b).

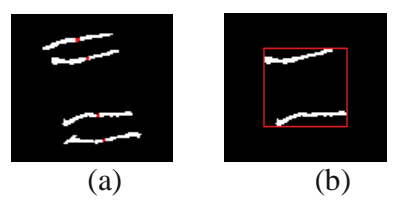

Fig 4. (a) 4 CC centroids. (b) Segmented candidate vertebra

The segmented CCs are skeletonized and further pruned to remove spurious edges. Each CC is pieced into half horizontally and we now have four pieces $\mathrm{CC}_{\mathrm{TL}}$ (T-top, L-left), $\mathrm{CC}_{\mathrm{TR}}$, $\mathrm{CC}_{\mathrm{BL}}$ and $\mathrm{CC}_{\mathrm{BR}}$. The reason for piecing is based on the fact that osteoporotic fractures are categorized into 3 cases along with specific morphological grades [19] and this manner of piecing helps to identify and confirm fractures than analyzing them as a whole.

A few steps of PCA are performed on each piece. The motivation to pick PCA is that it doesn't require the full shape to discover its direction. PCA is often used for dimensionality reduction than for angle estimation. PCA finds the direction along which our data varies the most. This is done by finding Eigen values of covariance matrix of the given data. Covariance matrix of the set of pixels $((\mathrm{x}, \mathrm{y})$ coordinates are the features) in a $\mathrm{CC}$ is found and the Eigen values identified. The Eigen vector corresponding to the largest Eigen value $\left(\mathrm{EV}_{\mathrm{L}}\right)$ is the direction of the largest variation. Inclination of this direction with $\mathrm{X}$-axis is found using (6) and the angle is estimated as in (7).

$$
\begin{aligned}
& \mathrm{D}=\cos ^{-1}\left(\mathrm{EV}_{\mathrm{L}}\right) \\
& \theta=\mathrm{D} * \frac{180}{\pi}
\end{aligned}
$$


(c)

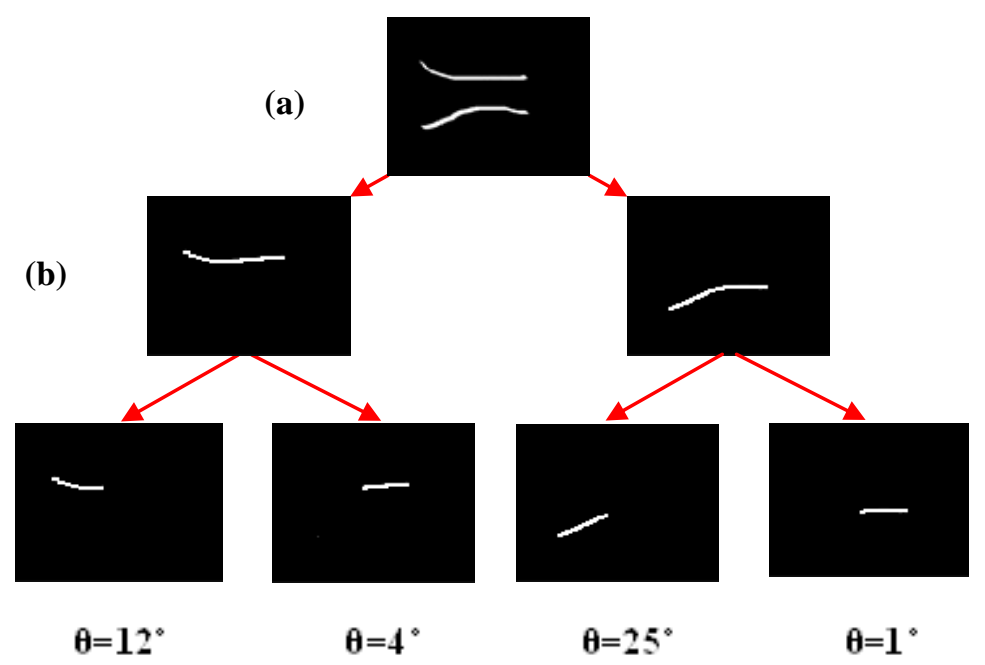

Fig 5. Illustrations (a) Segmented vertebrae (b) Superior $\left(\mathrm{CC}_{\mathrm{T}}\right)$ and inferior $\left(\mathrm{CC}_{\mathrm{B}}\right)$ endplates

(c) Four individual pieces of the endplate $\left(\mathrm{CC}_{\mathrm{TL}}\right),\left(\mathrm{CC}_{\mathrm{TR}}\right),\left(\mathrm{CC}_{\mathrm{BL}}\right)$ and $\left(\mathrm{CC}_{\mathrm{BR}}\right)$ for independent PCA angle estimation (d) Estimated angles using (7)

We use (8) to classify if the estimated angle of each endplate piece resulted in a normal or fractured vertebral piece.

$$
\text { Normal }\left\{\begin{array}{l}
\boldsymbol{\theta} \leq \mathrm{T}_{\mathrm{h} 1} \text {, when the slope of axis of maximum variation is +ve } \\
\boldsymbol{\theta} \geq \mathrm{T}_{\mathrm{h} 2} \text {, when the slope of axis of maximum variation is -ve }
\end{array}\right.
$$

Fractured Otherwise

All four PCA angles resulting from two endplates and each with two pieces are used in the final classification of normal or fractured vertebra. If one or more of the angles measured point to a fracture, the final classification is a fractured vertebra.

\section{EXPERIMENTAL RESULTS}

The dataset was from MICCAI 2016 challenge 'Automatic Vertebral Fracture Analysis and Identification from VFA by DXA' which consisted of thoracolumbar spine acquired at a resolution of between 1 and $0.35 \mathrm{~mm}$ with Hologic Discovery A DXA scanner using the Instant Vertebral Assessment (IVA) scan option. Each visible vertebra in the image was from T5 to L4. The proposed framework was applied to 527 DXA images of which 480 were normal and 47 were fractured. This yielded classification sensitivity of $89.36 \%$ and specificity of $94.37 \%$, with corresponding accuracy of $93.93 \%$ and an error of $6.07 \%$. The parameter that gave the best differentiation between normal and fractured vertebral bodies was the angle of inclination and concavity of both vertebral endplates set as $\leq 10^{\circ}$ and $\geq 170^{\circ}$ with $\theta$ bearing the degrees between $0-180$ in the experiments. The program execution was done using a PC with $2.93 \mathrm{GHz}$ Dual Core and 2 GB RAM.

Table 1 shows the confusion matrix as a result of classification. In table 2, details of sensitivity, specificity are given. Graph in figure 6 shows the True Positive Rate (TPR), True Negative Rate (TNR), False Positive Rate (FPR), False Negative Rate (FNR) for determination of fracture in each of the vertebral level T7 to L4. 
DOI: https://dx.doi.org/10.26808/rs.ca.i8v1.19

International Journal of Computer Application (2250-1797)

Volume 8-volume 1,January-February 2018

Table 1. Table of Confusion matrix

\begin{tabular}{|c|c|c|c|c|c|c|c|}
\hline $\begin{array}{c}\text { Actual } \\
\text { class } / \\
\begin{array}{c}\text { Predicted } \\
\text { class }\end{array}\end{array}$ & Fracture & $\begin{array}{l}\text { No } \\
\text { Fracture }\end{array}$ & Total & TPR $=\frac{T P}{T P+F N}$ & TNR $=\frac{T N}{T N+F P}$ & FPR $=\frac{F P}{F P+T N}$ & FNR $=\frac{F N}{F+T P}$ \\
\hline \begin{tabular}{c} 
Fracture \\
\cline { 3 - 4 } $\begin{array}{c}\text { No } \\
\text { Fracture }\end{array}$
\end{tabular} & 42 & 5 & 47 & $94.37 \%$ & $5.62 \%$ & $10.63 \%$ \\
\hline Total & 69 & 453 & 480 \\
\hline
\end{tabular}

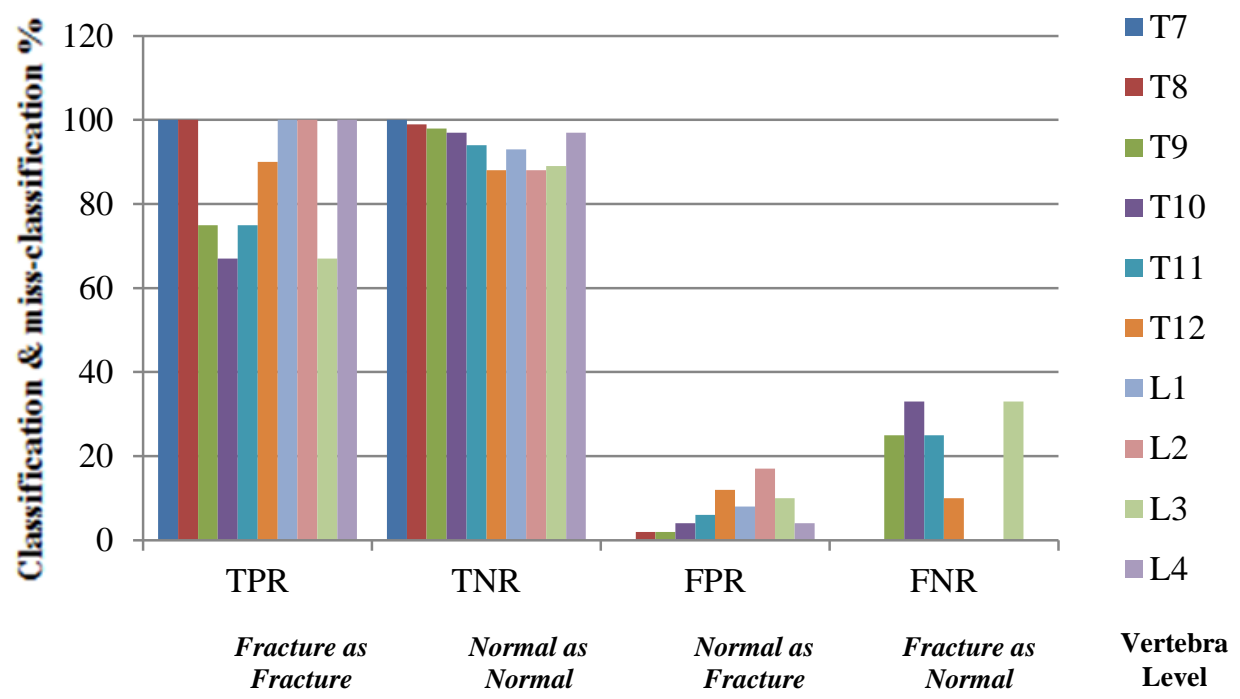

Fig 6. Per vertebra ratios of TP, TN, FP and FN

Our method fails only for those cases that have extremely varying noise intensities as shown in figure 2. Perhaps, these require multiple noise reducing filters acting on the same image.

\section{CONCLUSION}

Assisting radiologists is of profound importance as the number of images used for diagnosis of fractures is large and the imaging modalities used are on the rise. Reliable automatic and semi-automatic quantitative methods for determination of fractures can help reduce the load on radiologists and can serve as verification process. The proposed automatic method segments individual vertebra restricting its primary interest into segmenting only the endplates of the vertebra after being preprocessed to reduce the noise. The direction of the highest variation of a piece of an endplate is found as in PCA. Angle made by this direction with $\mathrm{X}$-axis indicates the inclination of the endplate piece, which is used to classify if the vertebra is normal or fractured. Desirable features of medical image segmentation and classification algorithms like accuracy, robustness, reliability, repeatability, and least dependency on operators have been followed to a large extent in this work. Our method does not require neighbor pixel information, no explicit shape priors, no templates, no user intervention, and can subsequently manage a wide variety of anatomical variations. The final outcomes demonstrate that reliably accurate segmentation and classification can be acquired on each of the different thoracic and lumbar vertebrae from diverse subjects. 
In future research, we plan to implement multiple noise reducing filters for a single image, evaluate our method to automatically classify fracture type and extend the methods to threedimensional analysis of vertebral bodies.

\section{REFERENCE}

[1] Gavriil Tsechpenakis, Deformable Model-Based Medical Image Segmentation, Multi Modality State-of-the-Art Medical Image Segmentation and Registration Methodologies, Volume 1, 2011, XII, 410 p, ISBN 978-1-4419-8194-3

[2] Ili Ayuni Mohd Ikhsan, Aini Hussain, Mohd Asyraf Zulkifley, Nooritawati Md. Tahir, Aouache Mustapha, An Analysis of X-Ray Image Enhancement Methods for Vertebral Bone Segmentation, 2014 IEEE 10th International Colloquium on Signal Processing \& its Applications

[3] Neeraj Sharma, Lalit M. Aggarwal, Automated Medical Image Segmentation Techniques, Journal of Medical Physics, 2010, 35(1): 3-14

[4] Adela Arpitha, Lalitha Rangarajan, Automatic Segmentation of Vertebral Body using Clustering and Geometrical Parameters, 2017 6th International Conference on Signal \& Image Processing. In Press

[5] MICCAI 2016 challenge, Automatic Vertebral Fracture Analysis and Identification from VFA by DXA

[6] Robert Korez, Bulat Ibragimov, Boštjan Likar, Franjo Pernuš and Tomaž Vrtovec, An Improved Shape-Constrained Deformable Model for Segmentation of Vertebrae from CT Lumbar Spine Images, Recent Advances in Computational Methods and Clinical Applications for Spine Imaging, Lecture Notes in Computational Vision and Biomechanics (2015)

[7] M. G. Roberts, T. Oh, E. M. B. Pacheco, R. Mohankumar, T. F. Cootes, J. E. Adams, Semi-automatic determination of detailed vertebral shape from lumbar radiographs using active appearance models, Osteoporos Int (2012) 23:655-664

[8] Jean Baptiste Courbot, Edmond Rust, Emmanuel Monfrini, Christophe Collet, 2-Step Robust Vertebra Segmentation, Image Processing Theory, Tools and Application, 2015 IEEE

[9] Roberts, M.G, Cootes, T.F, Adams, J.E, Automatic location of vertebrae on DXA images using random forest regression, MICCAI 2012, Part III. LNCS, vol. 7512, pp. 361-368.

[10] Roberts MG, Pacheco EM, Mohankumar R, Cootes TF, Adams JE, Detection of vertebral fractures in DXA VFA images using statistical models of appearance and a semi-automatic segmentation, (2010) Osteoporos Int. 21(12). 2037-2046.

[11] Tobias Klinder, Jörn Ostermann, Matthias Ehm, Astrid Franz, Reinhard Kneser, Cristian Lorenz, Automated model-based vertebra detection, identification, and segmentation in CT images, Med. Image Anal. 13(3), 471-482 (2009)

[12] Robert van der Velde, Takouhi Ozanian, Bianca Dumitrescu, Jane Haslam, Joes Staal, Alan Brett, Joop van den Bergh, Piet Geusens, Performance of statistical models of shape and appearance for semiautomatic segmentations of spinal vertebrae T4-L4 on digitized vertebral fracture assessment images, The Spine Journal 15 (2015) 1248-1254

[13] Ferrar L, Jiang G, Schousboe JT, DeBold CR, Eastell R, Algorithm-based qualitative and semiquantitative identification of prevalent vertebral fracture: agreement between different readers, imaging modalities, and diagnostic approaches, 2008, J Bone Miner Res. 23(3), 417-424

[14] Lindsay R, Silverman SL, Cooper C, Hanley DA, Barton I, Broy SB, Licata A, Benhamou L, Geusens P, Flowers K, Stracke H, Seeman E, Risk of new vertebral fracture in the year following a fracture, 2001 JAMA 285(3): 320-323 
[15] Martin G Roberts, Automatic Detection and Classification of Vertebral Fracture using Statistical Models of Appearance, 2008 Thesis

[16] Annotations Part II Vertebral Fracture Initiative. OInternational Osteoporosis Foundation, March 2011

[17] Delmas PD1, van de Langerijt L, Watts NB, Eastell R, Genant H, Grauer A, Cahall DL, Underdiagnosis of vertebral fractures is a worldwide problem: the IMPACT study. J Bone Miner Res. 2005 Apr;20(4):557-63. Epub 2004

[18] James F. Griffith, Identifying osteoporotic vertebral fracture, Quant Imaging Med Surg 2015; 5(4):592-602

[19] Harry K. Genant,I Chun Y. Wu, Cornelis Van Kuijk, Arid Michael C. Nevitt, Vertebral Fracture Assessment Using a Semiquatitative Technique, Journal Of Bone And Mineral Research Volume 8, Number 9, 1993 